\title{
Capacitação de trabalhadores em suporte básico de vida
}

\author{
Training workers in basic life support
}

\author{
Capacitación de trabajadores en soporte básico de vida
}

\author{
Maria João Filomena dos Santos Pinto Monteiro ${ }^{1}$, Maria da Conceição Alves Rainho Soares Pereira ${ }^{2} \oplus^{\circledR}$

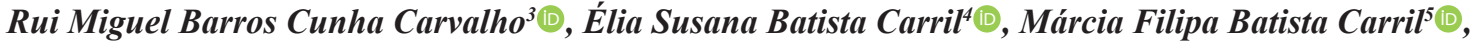 \\ Vitor Manuel Costa Pereira Rodrigues ${ }^{6}$
}

\section{Histórico \\ Recibido: \\ 01 de febrero de 2018 \\ Aceptado: \\ 10 de abril de 2018}

1 Doutora, Professora Coordenadora Centro de Investigação em Tecnologias e Serviços de Saúde, Universidade de Trás-os-Montes e Alto Douro, Escola Superior de Saúde, Vila Real, Portugal. Autor de Correspondência. E-mail: mjmonteiro@utad.pt

2 Doutora, Professora Coordenadora, Centro de Investigação em Desporto, Saúde e Desenvolvimento Humano, Universidade de Trásos-Montes e Alto Douro, Escola Superior de Saúde, Vila Real,

3 Mestre, Enfermeiro, Centro Hospitalar de Trás-os-Montes e Alto Douro, Vila Real, Portugal.

4 Mestre, Enfermeira, Centro Hospitalar de Trás-os-Montes e Alto Douro, Vila Real, Portugal.

5 Licenciada, Enfermeira, Unidade de Cuidados Continuados Integrados de Alijó, Alijó, Portugal.

6 Agregado, Professor Coordenador com Agregação, Centro de Investigação em Desporto, Saúde e Desenvolvimento Humano, Universidade de Trás-os-Montes e Alto Douro, Escola Superior de Saúde, Vila Real, Portugal.

\begin{abstract}
Resumo
Introdução: A capacitação dos cidadãos no auxílio a vítimas em risco de vida é fundamental em qualquer sociedade. Objetivo: avaliar os conhecimentos sobre Suporte Básico de Vida dos trabalhadores de uma Câmara Municipal do norte de Portugal, antes e após a implementação do programa de intervenção "Capacitar para Salvar" e avaliar as competências demonstradas, após a participação no programa de intervenção. Materiais e Métodos: Trata-se de um estudo do tipo quase-experimental e longitudinal, onde foi utilizado um questionário, constituído por 2 partes (caraterização sociodemográfica e avaliação de conhecimentos) e uma grelha de avaliação de competências. No $1^{\circ}$ momento foram avaliados os conhecimentos sobre Suporte Básico de Vida e implementado um programa de intervenção, sendo que no $2^{\circ}$ momento, foram avaliados os conhecimentos e competências sobre Suporte Básico de Vida. Resultados: Participaram no estudo 97 trabalhadores. Os resultados mostraram que os trabalhadores possuem conhecimentos e competências insuficientes em Suporte Básico de Vida. Discussão e Conclusões: Verificaram-se diferenças estatisticamente significativas nos conhecimentos e competências em Suporte Básico de Vida. Implicações para a prática: implementação de estratégias que promovam a formação em Suporte Básico de Vida a todos cidadãos, quer ao nível da transmissão de conhecimentos, quer da formação prática.
\end{abstract}

Palavas chave: Suporte Básico de Vida; Capacitação; Trabalhadores.

Abstract

Introduction: Training citizens to help victims in life-threatening situations is fundamental in any society. Objective: to evaluate the knowledge on Basic Life Support by workers in a Municipal Council of the north of Portugal, before and after implementing the intervention program "Train to Save" and assess the skills shown after participating in the intervention program. Materials and Methods: This was a quasi-experimental longitudinal study, using a questionnaire, which comprised two parts (sociodemographic characterization and evaluation of knowledge) and a skills-assessment grid. In the first moment, knowledge on Basic Life Support was evaluated and an intervention program was implemented; the second moment evaluated the knowledge and skills on Basic Life Support. Results: The study had the participation of 97 workers. The results revealed that the workers had insufficient knowledge and skills in Basic Life Support. Discussion and Conclusions: Statistically significant differences were verified of knowledge and skills in Basic Life Support. Implications for the practice: implementation of strategies that promote training in Basic Life Support for all citizens at knowledge transmission level and in practical formation.

Key words: Cardiopulmonary Resuscitation; Training; Workers.

Resumen

Introducción: La capacitación de los ciudadanos en la ayuda a las víctimas en riesgo de vida es fundamental en cualquier sociedad. Objetivo: evaluar los conocimientos sobre Soporte Básico de Vida de los trabajadores de una Cámara Municipal del Norte de Portugal, antes y después de la implementación del programa de intervención "Capacitar para Salvar" y evaluar las competencias demostradas, después de la participación en el programa de intervención. Materiales y Métodos: Se trata de un estudio de tipo cuasi-experimental y longitudinal, donde se utilizó un cuestionario, constituido por 2 partes (caracterización sociodemográfica y evaluación de conocimientos) y una plantilla de evaluación de competencias. En el $1^{\circ}$ momento fueron evaluados los conocimientos sobre Soporte Básico de Vida e implementado un programa de intervención, siendo que en el $2^{\circ}$ momento fueron evaluados los conocimientos y competencias sobre Soporte Básico de Vida. Resultados: Participaron en el estudio 97 trabajadores. Los resultados mostraron que los trabajadores poseen conocimientos y competencias insuficientes en Soporte Básico de Vida. Discusión y Conclusiones: Se verificaron diferencias estadísticamente significativas en los conocimientos y competencias en Soporte Básico de Vida. Implicaciones para la práctica: implementación de estrategias que promuevan la formación en Soporte Básico de Vida a todos los ciudadanos, tanto en el nivel de la transmisión de conocimientos como en la formación práctica.

Palabras clave: Reanimación Cardiopulmonar; Capacitación; Trabajadores.

Como citar este artigo: Monteiro MJFSP, Pereira MCARS, Carvalho RMBC, Carril ÉSB, Carril MFB, Rodrigues VMCP. Capacitação de trabalhadores em suporte básico de vida. Rev Cuid. 2018; 9(2): 2117-26. http://dx.doi.org/10.15649/cuidarte.v9i2.505

(c) (1) () (C2018 Universidad de Santander. Este es un artículo de acceso abierto, distribuido bajo los términos de la licencia Creative Commons Attribution (CC BY-NC 4.0), que permite el uso ilimitado, distribución y reproducción en cualquier medio, siempre que el autor original y la fuente sean debidamente citados. 


\section{INTRODUÇÃO}

A educação para a cidadania tem como principal objetivo possibilitar a aquisição de conhecimentos e competências para a inserção responsável dos cidadãos e que pode ser assumida sob a forma de elaboração e desenvolvimento de projetos, em parceria entre as instituições académicas, sector empresarial e a comunidade em geral $^{1}$. Neste sentido o desenvolvimento de competências deve ser entendido como a capacidade para uma determinada tarefa no sentido de resolver uma situação e pressupõe, antes de tudo, a aquisição de conhecimentos. Possuir conhecimentos e habilidades para prestar auxílio de forma adequada e sistemática a um qualquer cidadão que dele necessita pode representar a diferença entre a vida e a morte de um ser humano. É portanto de vital importância a obtenção de conhecimentos em Suporte Básico de Vida. Conforme refere Martins" ${ }^{2}$ " "nesta partilha de responsabilidades cada cidadão deve ser um participante ativo da sua segurança e da segurança coletiva" e "cada um de nós tem o dever cívico de desenvolver uma verdadeira apetência de preparação face ao perigo. Qualquer que seja o ambiente onde o cidadão está inserido, este tem em si a responsabilidade de prestar toda a assistência para o qual está capaz e lhe seja possível. Apesar de existirem diversas definições de Suporte Básico de Vida constata-se um registo homogéneo nos seus significados, procurandose o recurso a fontes mais atuais e pertinentes. Por exemplo, em 2010³, Suporte Básico de Vida é definido como "medidas iniciais e imediatas aplicadas à vítima, fora do ambiente hospitalar, onde são realizadas ações por pessoal treinado na tentativa de manter os sinais vitais - respiração, pulsação, temperatura e pressão arterial -, evitando o agravamento de lesões". Rocha ${ }^{4}$, refere que Suporte Básico de Vida compreende o atendimento prestado a uma vítima de mal súbito ou trauma, visando a manutenção de seus sinais vitais e a preservação da vida, além de evitar o agravamento das lesões existentes, até que uma equipa especializada possa transportá-la ao hospital e oferecer um tratamento definitivo.

O Instituto Nacional de Emergência Médica refere-se ao Suporte Básico de Vida, como um conjunto de medidas e procedimentos técnicos, que permitem ganhar tempo, mantendo alguma circulação e ventilação na vítima até à chegada de um socorro mais diferenciado, capaz de instituir procedimentos de Suporte Avançado de Vida ${ }^{5}$. Das definições apresentadas todas convergem para apresentar o Suporte Básico de Vida como um conjunto de técnicas e procedimentos, considerando o primeiro atendimento a ser implementado em vítimas que estejam a correr risco de vida, podendo recebê-los na rua, em ambiente doméstico ou profissional. Quanto aos objetivos do Suporte Básico de Vida são enumerados a preservação das condições vitais da vítima e do seu transporte sem causar traumas ou danos ocorridos durante a manipulação e remoção inadequada. O socorrista deve ter como princípio básico evitar o agravamento das lesões e procurar estabilizar, de forma imediata, as funções respiratórias e hemodinâmicas da vítima ${ }^{4}$. As manobras de Suporte Básico de Vida são susceptiveis de ser aprendidas por qualquer pessoa. Estas, devem ser iniciadas o mais precocemente e rapidamente possível no local onde ocorreu o acidente, desde que existam condições de segurança, quer para a vítima quer para o prestador de cuidados. A variável tempo assume importância incontestável no que respeita ao sucesso dareanimaçãocardiorrespiratória. Uma rápida intervenção na realização das manobras de Suporte Básico de Vida aumenta a probabilidade de sobrevivência da vítima, desde que as pessoas 
que as praticam as saibam realizar de forma eficiente e eficaz. O Suporte Básico de Vida integra um dos quatro elos que compõem a cadeia de sobrevivência. A cadeia de sobrevivência, em geral, é referida como um conjunto de ações que relacionam a vítima de paragem cardíaca súbita com a sobrevida ${ }^{6}$. A cadeia de sobrevivência representa, simbolicamente, o conjunto de procedimentos que permitem salvar vítimas de paragem cardio-respiratória ${ }^{7-8}$. Consideram-se três atitudes fundamentais que modificam o resultado no socorro à vítima que se encontra em paragem cardio-respiratória: i) pedir ajuda, acionando de imediato o Sistema Integrado de Emergência Médica; ii) iniciar de imediato manobras de Suporte Básico de Vida. Estas compreendem um conjunto de etapas e procedimentos sequenciais que devem ser colocados em prática para que este seja bem-sucedido. Incluem as seguintes etapas: avaliação inicial; manutenção de via aérea permeável e compressões torácicas e ventilação com ar expirado. Esta sequência de procedimentos, após a avaliação inicial, segue as etapas ' $\mathrm{ABC}$ ', com as cujas iniciais provêm dos termos ingleses airway, breathing e circulation; iii) aceder à desfibrilhação tão precocemente quanto possível mas apenas quando indicado. Estes procedimentos ocorrem de forma encadeada sucedendo-se de forma sistemática e constituem uma cadeia de atitudes representadas por elos. Cada elo articula o procedimento anterior com o seguinte. Neste âmbito, surge o conceito de cadeia de sobrevivência, composta por quatro elos ou ações criteriosamente definidos ${ }^{7}$ : i) acesso precoce ao Sistema Integrado de Emergência Médica; ii) início precoce do suporte básico de vida; iii) desfibrilhação precoce e iv) suporte avançado de vida precoce. Se tivermos em conta que a principal causa de morte no espaço europeu é a paragem cardiorrespiratória súbita ${ }^{9}$, e que dois terços destas ocorrências se passam em ambiente extra-hospitalar, compreenderemos que é fundamental que qualquer cidadão esteja apto a iniciar e exercer de forma correta manobras de Suporte Básico de Vida. Em Portugal, são também as doenças cardiovasculares que constituem um dos problemas mais graves para a população e a maior parte das mortes evitáveis associa-se à doença coronária, ocorrendo em contexto extrahospitalar ${ }^{7}$. Atendendo a toda esta problemática, foi desenvolvido um estudo com o objetivo de avaliar os conhecimentos sobre Suporte Básico de Vida dos trabalhadores de uma Câmara Municipal, antes e após a implementação do programa de intervenção "Capacitar para Salvar" e avaliar as competências demonstradas em Suporte Básico de Vida, após a participação no programa de intervenção.

\section{MATERIAIS E MÉTODOS}

Trata-se de um estudo de natureza quantitativa, enquadrando um estudo do tipo quaseexperimental e longitudinal. $\mathrm{O}$ carácter longitudinal é justificado pela intenção de elaborar um processo de recolha de dados em dois momentos junto do mesmo grupo. Fortin, Côté e Fillion ${ }^{10}$, referem que "o estudo longitudinal recolhe dados de forma periódica junto dos mesmos grupos ... e começa no presente e termina no futuro", sendo que a tipologia do estudo referida, caracteriza-se pela ausência de um grupo de controlo ou repartição aleatória e a existência de uma variável manipulável. $\mathrm{O}$ presente estudo integrou três fases que, sumariamente, se descrevem:

i) a primeira fase compreendeu a avaliação inicial dos conhecimentos sobre Suporte Básico de Vida dos trabalhadores de uma Câmara Municipal, através do preenchimento de um questionário e decorreu entre março e abril de 2016; 
ii) a segunda fase constou essencialmente da implementação de um programa de intervenção "Capacitar para Salvar", com quatro sessões com enfâse na tipologia teórico-prática sobre Suporte Básico de Vida. Cada sessão teve a duração de duas horas e decorreu durante o mês de abril de 2016;

iii) A terceira fase diz respeito à avaliação do programa de intervenção: três meses após a implementação do programa de intervenção "Capacitar para Salvar" para avaliação dos conhecimentos e competências em Suporte Básico de Vida. Utilizou-se o questionário inicial para avaliação de conhecimentos e uma grelha de observação para avaliação de competências. Esta fase decorreu durante os meses de junho e julho de 2016.

A população que esteve na base deste estudo foi constituida por todos os trabalhadores de uma Câmara Municipal do norte de Portugal. Foi definido como critério de exclusão os trabalhdores que tiveram formação sobre Suporte Básico de Vida há menos de dois anos. A amostra, foi selecionada com base numa amostragem não probabilística por conveniência, sendo integrada por todos os trabalhadores que manifestaram o seu consentimento para participar no estudo. Como instrumentos de recolha de dados foram utilizados:

i) um questionário constituido por 2 partes: uma de caraterização sociodemográfica e profissional com 8 questões e uma segunda parte constituida por um questionário de avaliação de conhecimentos sobre Suporte Básico de Vida no que respeita a princípios gerais e objetivos sobre Suporte Básico de Vida, abordagem de uma vítima adulta, início das manobras de reanimação, relação entre compressões/ventilações, modo de realização das compressões, posição lateral de segurança, avaliação da respiração espontânea, manutenção de Suporte Básico de Vida e ventilação boca a boca. Trata-se de um questionário composto por um conjunto de 13 afirmações, em relação às quais o trabalhador assinalava a afirmação que melhor traduzia a sua opinião;

ii) uma grelha de avaliação de competências em Suporte Básico de Vida, onde era registada a competência demonstrada ou não demonstrada, relativamente a 6 parâmetros: avaliação de condições de segurança, avaliar se vítima responde, pedido de ajuda, permeabilização da via aérea, avaliação da respiração e execução de compressões torácicas.

O estudo foi autorizado pela Comissão de Ética da Universidade de Trás-os-Montes e Alto Douro (parecer 11/2016) e as pessoas envolvidas foram informadas e esclarecidas acerca da natureza e objectivos do estudo e convidadas a participar de forma voluntária, sabendo que não existiam riscos nem desconfortos para quem participasse no estudo, sendo assegurado o total anonimato e confidencialidade das informações obtidas.

Os dados recolhidos foram introduzidos e tratados no software aplicativo IBM $® S P S S$ V22. Foi realizada a análise descritiva dos dados recorrendo à estatística descritiva e inferencial das observações recolhidas. Foi verificada para as variáveis contínuas utilizadas, a ausência de uma distribuição normal $(\mathrm{p}<0,05)$, das respostas dadas pelos participantes. A verificação foi realizada com recurso ao teste KolmogorovSmirnov (K-S) que serve para analisar a aderência à normalidade da distribuição de uma variável. O teste pressupõe a rejeição da normalidade da distribuição dos dados se o níivel de significância for inferior a $5 \%(p<0,05)$. Como não se 
verificou uma distribuição normal, optou-se pela utilização de testes não paramétricos para a estatística inferencial.

\section{RESULTADOS}

A amostra é constituída por 97 trabalhadores, $74,2 \%$ do sexo feminino e $25,8 \%$ do masculino. A idade dos inquiridos, varia entre um mínimo de 23 anos e um máximo de 66 anos, sendo a média de idade de 44,2 anos. No que respeita ao estado civil, a maioria $(57,7 \%)$ dos inquiridos são casados, $25,8 \%$ são solteiros e $10,3 \%$ são divorciados. Quanto ao nível de instrução, verificou-se que a maioria $(54,6 \%)$ dos participantes possui o ensino secundário, $29,9 \%$ o ensino superior e $12,4 \%$ o $3^{\circ}$ ciclo do ensino básico. No que diz respeito ao tempo de serviço, a maioria $(38,2 \%)$ dos inquiridos exerce funções naquele municipio entre 11 e 20 anos e 33,9\% exerce funções há 10 ou menos anos. Relativamente à área de residência, constata-se que $64,9 \%$ dos participantes do estudo residem em zona urbana, e os restantes 35,1\% em zona rural.

Atendendo à avaliação dos conhecimentos sobre Suporte Básico de Vida dos trabalhadores de uma Câmara Municipal do norte de Portugal, antes e após a participação no programa de intervenção "Capacitar para salvar", a Tabela 1 apresenta os resultados relativos às respostas corretas e incorretas no $1^{\circ}$ e $2^{\circ}$ momento para cada um dos itens que constam do questionário de Avaliação de Conhecimentos em Suporte Básico de Vida.
Relativamente aos itens com maior percentagem de respostas corretas destacam-se o item 10 "indique qual o número europeu de emergência médica:" com 94,8\%; o item 2 “o Suporte Básico de Vida tem por objetivo:" com 43,3\%; o item 7 "em que situação se deve colocar a vítima em posição lateral de segurança: “ com 40,2\% e o item 1 "os princípios gerais do Suporte Básico de Vida são:" com 38,1\%. Salienta-se que apenas 8,2\% dos participantes conhece como as compressões no tórax devem ser feitas (item 6) e $7,2 \%$ como proceder perante um adulto consciente com obstrução de via aérea (item 9).

No que concerne ao $2^{\mathrm{o}}$ momento, os dados demonstram que o maior número de respostas corretamente assinaladas, diz respeito aos itens 10 "indique qual o número europeu de emergência médica:" com 97,9\%; ao item 5 "nas manobras de reanimação a relação compressões/ ventilações na vítima adulta inconsciente e em paragem cardiorrespiratória é:" 90,7\%; ao item 3 "como deve proceder antes de abordar uma vítima adulta:" com $82,5 \%$ e ao item 8 "a avaliação da respiração espontânea e eficaz deve ser efetuada durante:" com 80,0\%. Os itens com menor número de respostas assinaladas são o item 1 "os princípios gerais do Suporte Básico de Vida são:" com $23,7 \%$ e o item 9 "como proceder perante um adulto consciente com obstrução grave de via aérea (asfixia) por corpo estranho (alimentos ou objetos):" com 24,7\%. Podemos, então, considerar que existiu um aumento do número de respostas corretamente assinaladas sobre os conhecimentos em Suporte Básico de Vida, com exceção para o item 1 "os princípios gerais do suporte básico de vida são" para a qual se observa um decréscimo de repostas corretamente assinaladas do $1^{\mathrm{o}}$ para o $2^{\circ}$ momento (37 vs 24 ). 
Tabela 1. Número, percentagem de respostas corretas e incorretas e nível de significância (teste de McNemar) ao questionário de avaliação de conhecimentos em Suporte Básico de Vida (antes e após a implementação do programa de intervenção). Norte de Portugal, 2016

\begin{tabular}{|c|c|c|c|c|c|c|c|c|c|}
\hline \multirow{3}{*}{$\begin{array}{l}\text { Itens de avaliação de } \\
\text { conhecimentos em } \\
\text { Suporte Básico de Vida }\end{array}$} & \multicolumn{4}{|c|}{$\mathbf{1}^{\mathbf{0}}$ Momento (antes) } & \multicolumn{5}{|c|}{$2^{\circ}$ Momento (após) } \\
\hline & \multicolumn{2}{|c|}{$\begin{array}{c}\text { Respostas } \\
\text { corretas }\end{array}$} & \multicolumn{2}{|c|}{$\begin{array}{l}\text { Respostas } \\
\text { incorretas }\end{array}$} & \multicolumn{2}{|c|}{$\begin{array}{c}\text { Respostas } \\
\text { corretas }\end{array}$} & \multicolumn{2}{|c|}{$\begin{array}{l}\text { Respostas } \\
\text { incorretas }\end{array}$} & \multirow{2}{*}{$\begin{array}{c}\begin{array}{c}\text { Teste } \\
\text { de } \\
\text { McNemar }\end{array} \\
\text { p }\end{array}$} \\
\hline & $\mathbf{N}$ & $\%$ & $\mathbf{N}$ & $\%$ & $\mathbf{N}$ & $\%$ & $\mathbf{N}$ & $\%$ & \\
\hline $\begin{array}{l}\text { 1. Os princípios gerais do } \\
\text { Suporte Básico de Vida são: }\end{array}$ & 37 & 38,1 & 60 & 61,9 & 23 & 23,7 & 74 & 76,3 & 0,022 \\
\hline $\begin{array}{l}\text { 2. O Suporte Básico de Vida } \\
\text { tem por objetivo: }\end{array}$ & 42 & 43,3 & 55 & 56,7 & 66 & 68 & 31 & 32 & $<0,001$ \\
\hline $\begin{array}{l}\text { 3. Como deve proceder antes } \\
\text { de abordar uma vítima adulta: }\end{array}$ & 32 & 33 & 65 & 67 & 80 & 82,5 & 17 & 17,5 & $<0,001$ \\
\hline $\begin{array}{l}\text { 4. Perante um adulto que não } \\
\text { respira, deve iniciar as } \\
\text { manobras de reanimação por }\end{array}$ & 11 & 11,3 & 86 & 88,7 & 69 & 71,1 & 28 & 28,9 & $<0,001$ \\
\hline $\begin{array}{l}\text { 5. A relação compressões } \\
\text { /ventilações na vítima } \\
\text { inconsciente e em paragem } \\
\text { cardiorrespiratória são: }\end{array}$ & 14 & 14,4 & 83 & 85,6 & 88 & 90,7 & 9 & 9,3 & $<0,001$ \\
\hline $\begin{array}{l}\text { 6. As compressões no tórax } \\
\text { devem ser feitas: }\end{array}$ & 8 & 8,2 & 89 & 91,8 & 30 & 30,9 & 67 & 36,1 & $<0,001$ \\
\hline $\begin{array}{l}\text { 7. Em que situação se deve } \\
\text { colocar a vítima em posição } \\
\text { lateral de segurança: }\end{array}$ & 39 & 40,2 & 58 & 59,8 & 76 & 78,4 & 21 & 21,6 & $<0,001$ \\
\hline $\begin{array}{l}\text { 8. A avaliação da respiração } \\
\text { espontânea e eficaz deve ser } \\
\text { efetuada durante: }\end{array}$ & 16 & 16,5 & 81 & 83,5 & 78 & 80,04 & 19 & 19,6 & $<0,001$ \\
\hline $\begin{array}{l}\text { 9. Como proceder perante } \\
\text { um adulto consciente com } \\
\text { obstrução de via aérea por } \\
\text { corpo estranho: }\end{array}$ & 7 & 7,2 & 90 & 92,8 & 24 & 24,7 & 73 & 75,3 & $<0,001$ \\
\hline $\begin{array}{l}\text { 10. Indique qual o número } \\
\text { Europeu de Emergência } \\
\text { Médica: }\end{array}$ & 92 & 94,8 & 5 & 5,2 & 95 & 97,9 & 2 & 2,1 & 0,375 \\
\hline $\begin{array}{l}\text { 11. Os quatros elos da cadeia } \\
\text { de sobrevivência são: }\end{array}$ & 33 & 34 & 64 & 66 & 67 & 69,1 & 30 & 30,9 & $<0,001$ \\
\hline $\begin{array}{l}\text { 12. O Suporte Básico de } \\
\text { Vida deve ser mantido sem } \\
\text { interrupção até: }\end{array}$ & 25 & 25,8 & 72 & 74,2 & 61 & 64,9 & 36 & 37,1 & $<0,001$ \\
\hline $\begin{array}{l}\text { 13. Durante a ventilação } \\
\text { boca a boca deve: }\end{array}$ & 23 & 23,7 & 74 & 76,3 & 65 & 67 & 32 & 33 & $<0,001$ \\
\hline
\end{tabular}

Relativamente à avaliação das competências demonstradas sobre Suporte Básico de Vida, após a participação no programa de intervenção
“Capacitar para Salvar”, estas foram sujeitas a verificação decorrido um período superior a dois meses após a participação no referido programa 
de intervenção. Procedeu-se à avaliação da execução prática e individual com registo numa grelha de avaliação de competências, essenciais para prestar socorro eficaz à vítima que se encontra em paragem cardio-respiratória. Os dados obtidos constam na Tabela 2 e demonstram que os itens executados corretamente por um maior número de indivíduos foram: o item
1 “avaliação de condições de segurança"e o item 3 "pedido de ajuda" ambos com 86 demonstrações corretas; o item 2 "avaliação da resposta da vítima" com 80, e o item 5 com 69 desmosntrações corretas. Contrariamente, o item 6 “execução de compressões torácicas” foi o que apresentou o menor número de participantes a executaram corretamente a técnica.

Tabela 2. Distribuição das frequências (absolutas e percentuais) de competências em Suporte Básico de Vida. Norte de Portugal, 2016

\begin{tabular}{|l|c|c|c|}
\hline Dimensão de competência & Competência & $\mathbf{N}$ & $\mathbf{\%}$ \\
\hline 1. Avaliação de condições de segurança & Não demonstrada & 11 & 11,3 \\
\hline 2. Avaliação de resposta da vítima & Demonstrada & 86 & 88,7 \\
\hline 3. Pedido de ajuda & Não demonstrada & 17 & 17,5 \\
& Demonstrada & 80 & 82,5 \\
\hline 4. Permeabilização da via aérea & Não demonstrada & 11 & 11,3 \\
\hline 5. Avaliação da respiração & Demonstrada & 86 & 88,7 \\
\hline 6. Execução de compressões torácicas & Não demonstrada & 41 & 42,3 \\
& Demonstrada & 56 & 57,7 \\
\hline 7. Execução de ventilações & Não demonstrada & 28 & 28,9 \\
& Demonstrada & 69 & 71,1 \\
\hline
\end{tabular}

\section{DISCUSSÃO}

Em Portugal a principal causa de morte é a paragem cardiorrespiratória súbita, com as doenças cardiovasculares a constituírem um dos problemas mais graves e a causa da maior parte das mortes evitáveis associadas à doença coronária. Estes factos tornam imperativa a promoção da capacitação dos cidadãos ditos "leigos", que este estudo releva como forma de intervir para aumentar os conhecimentos e habilidades da população para o atendimento precoce em situações de emergência e instituição do Suporte Básico de Vida como fundamental para salvar vidas.

Num estudo realizado em $2014^{11}$, com o objetivo de avaliar conhecimentos e competências em Suporte Básico de Vida em estudantes do $9^{\circ}$ e $12^{\circ}$ ano de escolaridade, encontraram-se resultados 
similares, com mais respostas corretamente assinaladas, nomeadamente, quanto à necessidade de asseguar as condições de segurança na abordagem à vítima $(99,6 \%)$ e que no nosso estudo foi de $82,5 \%$. Outro estudo ${ }^{12}$, corrobora o conhecimento que a população portuguesa adulta tem sobre a necessidade de avaliar as condições de segurança $(79,9 \%)$.

Pergola e Araujo ${ }^{13}$, ao identificarem os conhecimentos sobre Suporte Básico de Vida numa população leiga do Brasil, constataram que apenas 9,9\% conhece a manobra de respiração boca-a-boca, o que também é corroborado no presente estudo, onde apenas 23,7\% respondeu corretamente. Trata-se de um procedimento com alguma complexidade na medida em exige o cumprimento de alguns critérios específicos, como uma boa adaptação à boca da vítima e a hiperextensão do pescoço, no sentido de assegurar um fluxo uniforme de ar. Sobre o início das manobras de reanimação perante uma vítima que não respira, $88,7 \%$ não conhece o número de compressões que deve ser feito no tórax, resultados muito semelhantes aos do estudo da Cruz Vermelha Portuguesa ${ }^{14}$, com $71,3 \%$. Em relação às manobras de reanimação, a combinação da relação entre compressões/ ventilações nos estudos de Dixe e Gomes ${ }^{12}$, e no estudo de Rodrigues ${ }^{15}$, apontam para valores ligeiramente superiores quanto ao número de respostas corretas, respetivamente $20,2 \%$ e $44 \%$, em oposição aos resultados obtidos no presente estudo no $1^{\circ}$ momento $(14,4 \%)$ e que é amplamente superado no $2^{\circ}$ momento $(90,7 \%)$. $\mathrm{Na}$ explicitação da cadeia de sobrevivência, que consiste num conjunto ações que se sucedem de forma encadeada, a avaliação da respiração é determinante para a tomada de decisão sobre os procedimentos concretos e explícitos a seguir. Neste estudo, no $1^{\mathrm{o}}$ momento apenas $16,5 \%$ respondeu corretamente (10 segundos), verificando-se que no $2^{\circ}$ momento o valor percentual é consideravelmente mais elevado $(80,04 \%)$. Este resultado é muito similiar ao verificado no estudo de Dixe e Gomes ${ }^{12}$, ao referir que $77,9 \%$ dos participantes tinham intenção de o executar. O conhecimento específico sobre a posição lateral de segurança é fundamental para manter a permeabilidade da via aérea, e a sua execução deve ser feita de forma correta para evitar lesões adicionais na vítima. Verificou-se que apenas $40,2 \%$ detinha este conhecimento no $1^{\mathrm{o}}$ momento, valor superior $(30,8 \%)$ ao encontrado no estudo da Cruz Vermelha Portuguesa ${ }^{14}$. A obstrução da via aérea, normalmente como resultado de causas extrínsecas, é uma emergência que exige uma ação rápida e eficaz, sendo que apenas $7,2 \%$ e $24,7 \%$ responderam corretamente no $1^{\mathrm{o}}$ e $2^{\mathrm{o}}$ momento, respetivamente. $\mathrm{O}$ reduzido valor percentual das afirmações corretamente assinaladas pode dever-se à dificuldade em diferenciar se a obstrução é grave (asfixia) ou ligeira, o que também se verifica no estudo de Dixe e Gomes ${ }^{12}$.

De uma forma global e embora se tenham verificado competências em Suporte Básico de Vida, é necessário uma atenção especial para os procedimentos relativos à execução das compressões torácicas, sendo este procedimento determinante para assegurar em cerca de $25 \%$ o débito cardíaco. Apesar de reconhecermos que os conhecimentos são estruturantes para a 
execução correta, também temos consciência que o Suporte Básico de Vida exige destreza manual e competências muito específicas para o socorrista ser capaz de agir em situação concreta de prestação de auxílio a uma vítima em paragem cardio-respiratória. Decorre desta constatação a necessidade de periodicamente atualizar conhecimentos e melhorar as competências, através de projetos formativos que conjuguem uma dimensão mais teórica com uma dimensão prática em contexto de simulação. Esta constatação pode ser verificada num estudo realizado em $2014^{11}$, realizado com alunos do $9^{\circ}$ e $12^{\circ}$ ano de escolaridade, que, ao avaliarem as competências em três momentos diferentes, observaram que o registo sobre as competências melhora ao longo dos períodos de avaliação.

A reflexão sobre os resultados deste estudo orientam para a necessidade de continuar a formar e capacitar a população, em Suporte Básico de Vida, no sentido de conferir uma maior preparação para socorrer outras pessoas, assim como devemos ter em conta a responsabilidade social-empresarial no setor da saúde que cada instituição deve ter a seu cargo ${ }^{16}$, não esquecendo, a importância da promoção da saúde no local de trabalho que qualquer empresa, também, tem de $\operatorname{ter}^{17}$.

Como em qualquer investigação foram sentidas algumas limitações de entre as quais a impossibilidade de todos os colaboradores integrarem o programa de intervenção o que limitou o número de participantes e portanto a oportunidade de massificar a aquisição de conhecimentos e competências como estratégia fundamental para a melhoria das habilidades técnicas que integram o Suporte Básico de Vida.

\section{CONCLUSÕES}

Considerando os resultados obtidos neste estudo e no que concerne aos conhecimentos sobre Suporte Básico de Vida demonstrados, constatamos que a maioria dos participantes $(94,8 \%)$ já conhecia o número europeu de emergência médica antes da implementação do programa de intervenção. Nenhum dos itens avaliados que integram o questionário de avaliação de conhecimentos em Suporte Básico de Vida obteve 100\% de respostas corretas, o que permite concluir que os conhecimentos sobre Suporte Básico de Vida na população estudada são ainda insuficientes, estando em conformidade com alguns resultados relatados por outros estudos já apresentados.

Quanto à aquisição de conhecimentos sobre Suporte Básico de Vida entre $01^{\circ}$ e $\quad$ o $2^{\circ}$ momento, verificámos que existem diferenças estatisticamente significativas em todos os itens, com excepção do item 10, o que permite dizer que, após a implementação do programa de intervenção "Capacitar para salvar", os participantes no estudo adquiriram mais conhecimentos, o que releva o contributo positivo do mesmo, para a obtenção de ganhos em conhecimentos sobre Suporte Básico de Vida por parte dos trabalhadores de uma Câmara Municipal do norte de Portugal.

No que respeita às competências, a maioria dos indivíduos demonstrou saber realizar corretamente todas as manobras de Suporte Básico de Vida, com exceção da execução correta de compressões torácicas, na qual os participantes revelaram maiores dificuldades na sua concretização $(66,0 \%)$. Aliás, foi 
também neste domínio do conhecimento que ocorreu um elevado número de respostas não corretamente assinaláveis $(36,1 \%)$ mesmo após a implementação do programa de intervenção.

É um facto que a principal causa de morte no espaço europeu é a paragem cardiorrespiratória súbita. Este facto torna imperativa a promoção da capacitação dos cidadãos para que possam intervir com autonomia e segurança no auxílio a vítimas em risco de vida, devendo por isso possuir habilidades e conhecimentos adequados para prestar Suporte Básico de Vida de forma eficaz e eficiente. A reflexão sobre os resultados deste estudo, orienta para a necessidade de continuar a formar e capacitar a população em geral em Suporte Básico de Vida, no sentido de the conferir uma maior preparação para socorrer outras pessoas contribuindo para uma redução significativa das taxas de mortalidade e morbilidade o que se constitui num beneficio para a sociedade. A capacitação do cidadão dito "leigo" para o atendimento precoce em situações de emergência é fundamental para salvar vidas.

Conflito de interesses: Os autores declaram que não houve conflitos de interesse.

\section{REFERÊNCIAS}

1. Santos ME. Educação em cidadania/Educação pela cidadania/Educação para a cidadania. In Gonçalves $\mathrm{S}$, Sousa F (org). Escola e comunidade. Laboratórios de cidadania global. Instituto da Educação: Educa. 2012: 3954.

2. Martins G. Dinâmicas de Protecçao Civil. Revista Técnica e Formativa. Escola Nacional de Bombeiros. Lisboa, 2004, no 32.

3. Marchi JC, Nazário N. Suporte Básico de Vida. Livro Didático. Revista e atualizada. Palhoça, Unisul Virtual. 2010 .
4. Costa I, Nunes L, Ruivo A, Freitas A, Cerqueira A, Oliveira N. Manual de Suporte Básico de Vida e Desfibrilhação Automática Externa. Departamento de Enfermagem, Escola Superior de Saúde, Instituto Politécnico de Setúbal. 2012.

5. Instituto Nacional de Emergência Médica. Suporte Básico de Vida. Instituto Nacional de Emergência Médica. Edição. Lisboa, 2012.

6. Greif R, Lockey A, Conaghanc P, Lippert A, Vries W, Monsieurs K. European Resuscitation Council Guidelines for Resuscitation 2015. Section 10. Education and implementation of resuscitation. Resuscitation. 2015; (95): 288-301.

http://dx.doi.org/10.1016/j.resuscitation.2015.07.032

7. Instituto Nacional de Emergência Médica. Cadeia de Sobrevivência. Instituto Nacional de Emergência Médica. 2015.

8. Conselho Português de Ressuscitação. Versão Portuguesa das Recomendações 2010 para a Reanimação do European Resuscitation Council. Conselho Português de Ressuscitação. Porto, 2010.

9. Tavares A, Pedro N, Urbano J. Ausência de formacão em suporte básico de vida pelo cidadão: um problema de saúde pública? Qual a idade certa para iniciar? Revista Portuguesa de Saúde Pública. 2016; 34(1):101-4. https://doi.org/10.1016/j.rpsp.2015.06.006

10. Fortin MF, Côté J, Fillion F. Fundamentos e etapas do processo de investigação. Lusodidacta. Loures, 2009.

11. Gala CR. Competência dos alunos em SBV: Estudo comparativo entre o $9^{\circ}$ ano e $12^{\circ}$ ano de escolaridade. Dissertação de Mestrado. Instituto Superior Politécnico de Viseu. 2014.

12. Dixe MA, Gomes JC. Conhecimento da população portuguesa sobre Suporte Básico de Vida e disponibilidade para realizar formação. Revista da Escola de Enfermagem da Universidade de São Paulo. 2015; 49(4): 640-9. http://dx.doi.org/10.1590/S0080-623420150000400015

13. Pergola AM, Araujo I. O leigo e o suporte básico de vida. Revista da Escola de Enfermagem da Universidade de São Paulo. 2009; 43(2): 335-42. http://dx.doi.org/10.1590/S0080-62342009000200012

14. Cruz Vermelha Portuguesa. Portugal lidera falta de formação dos condutores em primeiros socorros. Cruz Vermelha Portuguesa, 2013.

15. Rodrigues VC. Conhecimentos da população do Concelho de Monção sobre Suporte Básico de Vida. Dissertação de Licenciatura. Universidade Fernando Pessoa. Porto, 2009.

16. Pérez-Ordoñez MC, Morales-Méndez JD. Revisión de los orígenes de la responsabilidad social empresarial en el sector salud en Colombia. Rev Cuid. 2011; 2(2): 206-15. http://dx.doi.org/10.15649/cuidarte.v2i1.59

17. Gómez ML, Laguado E. Propuesta de intervención de enfermería de los factores de riesgo que afectan un entorno laboral. Rev Cuid. 2013; 4(1): 557-63. http://dx.doi.org/10.15649/cuidarte.v4i1.18 